\title{
The Clinical Potential of Impella 5.0 Support in the Treatment of Recurrent Fulminant Viral Myocarditis with Profound Cardiogenic Shock
}

\author{
Shou Ogawa ${ }^{1}$, Makoto Suzuki ${ }^{1}$, Hiroyuki Ochi ${ }^{1}$, Mike Saji $^{1}$, Keitaro Mahara ${ }^{1}$, \\ Itaru Takamisawa ${ }^{1}$, Kosaku Nishigawa ${ }^{2}$, Yuko Furuichi ${ }^{3}$, \\ Shuichiro Takanashi ${ }^{2}$ and Mitsuaki Isobe ${ }^{1}$
}

\begin{abstract}
:
We herein report the clinical potential of Impella 5.0 support, which is a catheter-mounted micro-axial left ventricular support device, in a 39-year-old man with recurrent fulminant viral myocarditis complicated with profound cardiogenic shock despite inotropic infusion and an intra-aortic balloon pumping. Switching from these therapies to the Impella 5.0 device provided sufficient systemic perfusion with well-controlled left ventricular diastolic properties to facilitate a prompt recovery from profound cardiogenic shock. The patient was uneventfully discharged on the 27th hospital day. Given its effect of cardiac protection with sufficient systemic perfusion, the Impella device should be considered the first-line therapy for the treatment of fulminant myocarditis complicated with cardiogenic shock.
\end{abstract}

Key words: recurrent fulminant myocarditis, cardiogenic shock, Impella, left ventricular unloading

(Intern Med 58: 1459-1462, 2019)

(DOI: 10.2169/internalmedicine.1866-18)

\section{Introduction}

Fulminant myocarditis, defined as the presence of severe hemodynamic compromise requiring parenteral inotropic or mechanical circulatory support, carries an extremely high risk of fatal outcomes, accounting for $40 \%$ of in-hospital deaths in the acute phase $(1,2)$. Mechanical circulatory support using an intra-aortic balloon pumping and/or veno-atrial extracorporeal membrane oxygenation has been widely applied for cardiogenic shock due to fulminant myocarditis in Japan (3). In mid-2017, as an additional circulatory support device, the Impella device (Abiomed, Danvers, USA) (4), which is a catheter-mounted micro-axial left ventricular support device, was launched in Japan.

We herein report the clinical potential of Impella 5.0 (5.0 $1 /$ min) support in a 39-year-old man with recurrent fulminant viral myocarditis complicated with profound cariogenic shock despite inotropic infusion and intra-aortic balloon pumping. Our assessment of the serial hemodynamic profiles of the patient before and after Impella 5.0 support may highlight the clinical advantages of the Impella device in patients with this critical disorder.

\section{Case Report}

A 39-year-old man was referred to our institute for the evaluation of elevated plasma levels of cardiac enzymes with ST-segment elevation in his electrocardiogram.

Two weeks earlier, the patient had been treated for vomiting and diarrhea with a diagnosis of viral enteritis by his medical practitioner. Three days before admission to our institute, the patient was admitted to another hospital for the treatment of a sustained low-grade fever and epigastralgia.

On admission to our institute, his temperature was $36.5^{\circ} \mathrm{C}$, and his blood pressure was $95 / 69 \mathrm{mmHg}$ with a regular

${ }^{1}$ Department of Cardiology, Sakakibara Heart Institute, Japan, ${ }^{2}$ Department of Cardiovascular Surgery, Sakakibara Heart Institute, Japan and ${ }^{3}$ Department of Anesthesiology, Sakakibara Heart Institute, Japan

Received: July 23, 2018; Accepted: September 24, 2018; Advance Publication by J-STAGE: January 10, 2019

Correspondence to Dr. Makoto Suzuki, msuzuki@shi.heart.or.jp 

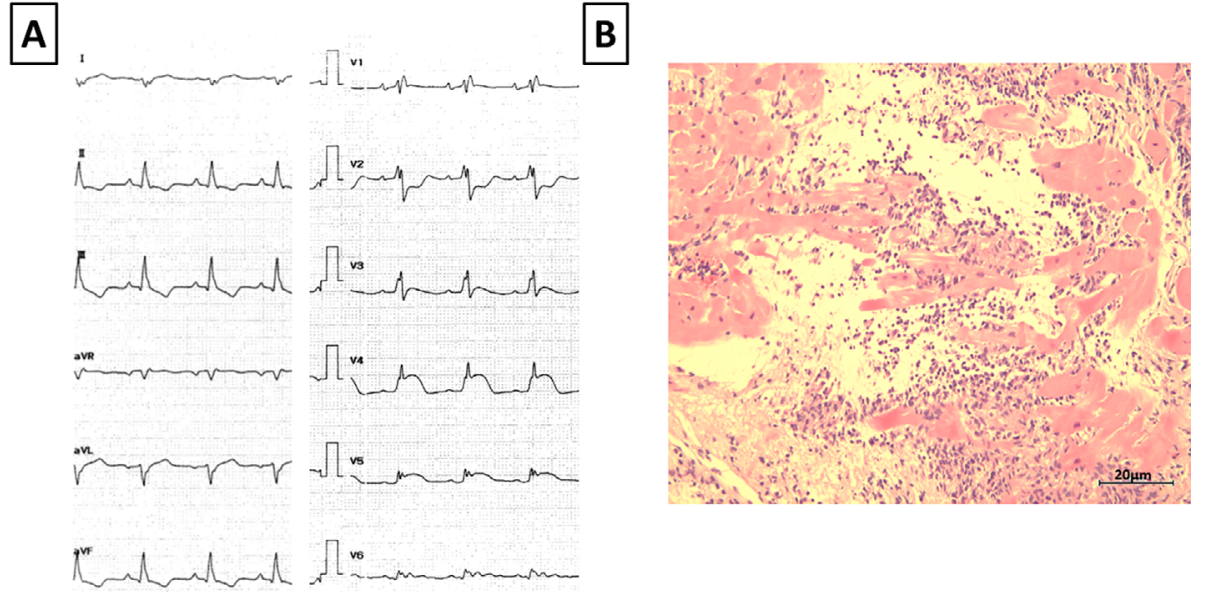

Figure 1. A 12-lead electrocardiogram (A) and endomyocardial-biopsy specimens (B) on admission.

pulse rate of 86/minute. A 12-lead electrocardiogram showed the first-degree atrial-ventricular block and rightbundle-branch-block with ST segment elevation in the left precordial leads (Fig. 1A), and an echocardiogram revealed an ejection fraction of $20 \%$ with diffuse left ventricular hypokinesis. The plasma levels of creatine kinase were 1,002 $\mathrm{U} / \mathrm{L}$ (normal range, 30-200), and troponin-T was $4.86 \mathrm{ng} /$ $\mathrm{mL}$ (normal <0.014). The patient had a history of intensive treatments for fulminant viral myocarditis in our institute five years earlier. An endomyocardial biopsy on admission revealed broad infiltrating lymphocytes and myocytolysis (Fig. 1B), resulting in a diagnosis of recurrent fulminant viral myocarditis.

The patient was started on a low dose of dobutamine on admission, followed by the addition of norepinephrine with intra-aortic balloon pumping the next day. Despite these intensive therapies, the cardiac power output, which equated to the cardiac external work per minute and was calculated as the product of the simultaneous cardiac output and mean arterial pressure (5), demonstrated a gradual decrease from 0.5 to 0.3 watts with a steep increase in atrial lactate levels from 1.6 to $5.1 \mathrm{mmol} / \mathrm{L}$. As indices of left ventricular diastolic properties, the diastolic pulmonary artery pressure (substitute for the mean left atrial pressure) and mean right atrial pressure were increased by over $20 \mathrm{mmHg}$.

Under a diagnosis of fulminant viral myocarditis with profound cardiogenic shock, switching from those therapies to the Impella 5.0 device was performed on the third hospital day (Fig. 2A and B). Impella 5.0 support rapidly increased the cardiac power output to 0.8 watts with a prompt normalization of atrial lactate levels and well-controlled diastolic pulmonary artery pressure and mean right atrial pressure (Fig. 2C). The addition of intravenous immunoglobulin treatment $(1.5 \mathrm{~g} / \mathrm{kg} \times 2$ days $)$ with Impella 5.0 support was also decided by our heart team. Impella 5.0 support was gradually terminated with stabilized hemodynamics and the normalization of plasma levels of cardiac enzymes on day 7 . Two days before the patient was discharged, a high neutral- izing antibody titer of 4,096 against Echovirus was detected. The patient ultimately fully recovered and was discharged from our institute on the 27th hospital day with a final left ventricular ejection fraction of $36 \%$ with the administration of beta-blocker and angiotensin-converting enzyme inhibitor.

\section{Discussion}

We report the dramatic hemodynamic profiles after switching from intra-aortic balloon pumping with inotropic infusion to Impella 5.0 support in a patient with recurrent fulminant viral myocarditis with profound cardiogenic shock. The present case may imply the clinical advantages of the early adoption of an Impella 5.0 device in patients with this critical disorder.

Three different types of mechanical circulatory support devices have been available to support acute hemodynamic compromise in Japan since mid-2017: intra-aortic balloon pumping, veno-atrial extracorporeal membrane oxygenation, and the Impella device. As shown in Fig. 2B, the Impella device is positioned across the aortic valve (cannula) to work as a micro-axial rotary pump (motor housing), expelling aspirated blood from the left ventricle (inlet area) to the ascending aorta (outlet area) under the automated Impella controller (differential pressure sensor) $(4,6)$. Veno-atrial extracorporeal membrane oxygenation has been utilized as a mechanical circulatory pump with the capacity to provide sufficient cardiac output with systemic oxygenation. However, this method of support increases the left ventricular oxygen demand due to an increase in the left ventricular afterload and this sometimes causes left ventricular thrombosis due to the inhibition of the ejection blood flow (7). In contrast, the Impella device increases the systemic circulatory perfusion by decreasing the left ventricular work, known as left ventricular unloading, thereby ameliorating the myocardial oxygen supply/demand ratio and improving the myocardial mechanical efficacy (8). At the first episode of fulminant viral myocarditis in the present case, circulatory sup- 

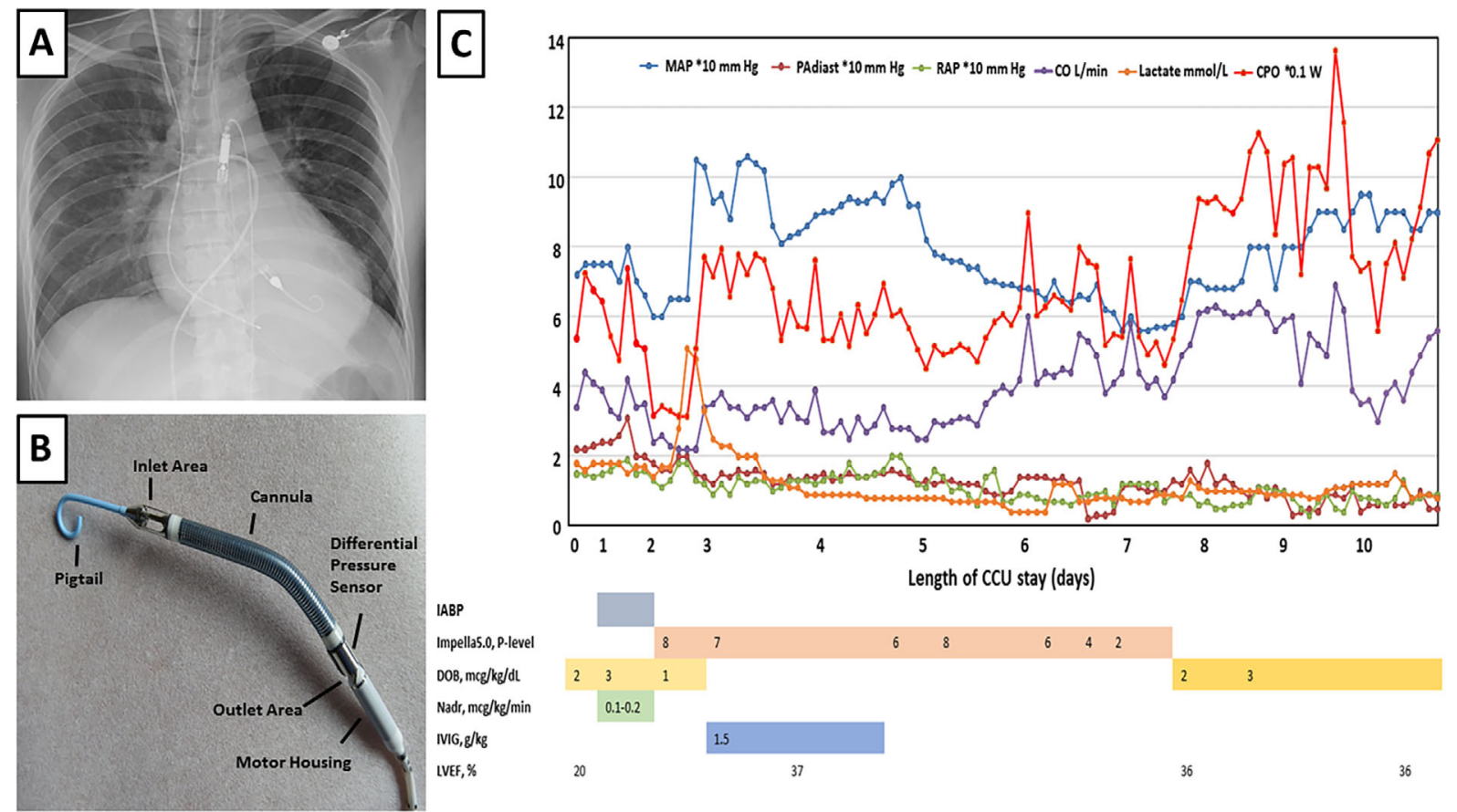

Figure 2. A: Chest X-ray findings with the deployment of the Impella 5.0 device. B: The composition of the Impella 5.0 device in the patient. C: Hemodynamic profiles before and after Impella 5.0 support. MAP: mean arterial pressure, PAdiast: diastolic pulmonary atrial pressure, RAP: mean right atrial pressure, CO: cardiac output, CPO: cardiac power output (calculated as MAP $\times$ CO/451), CCU: cardiac care unit, IABP: intra-aortic balloon pumping, Impella 5.0 P-level: 0 to 9 (highest number indicating maximal support), DOB: dobutamine, Nadr: noradrenaline, mcg: microgram, IVIG: intravenous immunoglobulin, LVEF: left ventricular ejection fraction

port using veno-atrial extracorporeal membrane oxygenation with intra-aortic balloon pumping required a large amount of blood products: 46 units of red blood cell transfusion, 29 units of fresh-frozen plasma transfusion, and 100 units of platelet transfusion with continuous hemodiafiltration. At present, with Impella 5.0 support, only 8 units of red blood cell transfusion and 6 units of fresh-frozen plasma transfusion with no need for continuous hemodiafiltration have been used.

The cardiac power output, which is reportedly the strongest index for predicting in-hospital mortality in cases of cardiogenic shock with a cut-off value of 0.53 watts (5), was extremely useful for assessing the serial hemodynamics in the present case. The value was improved temporarily after the initiation of inotropic therapy on admission, followed intra-aortic balloon pumping; however, it worsened immediately afterwards, dropping far below the cut-off value. Impella 5.0 promptly improved the cardiac power output while keeping all other hemodynamic parameters stable without the need for any inotropic support. While both Impella 2.5 (2.5 L/min) and Impella $5.0(5.0 \mathrm{~L} / \mathrm{min})$ are available in Japan in 2018, we chose Impella 5.0 because the power of Impella 2.5 was insufficient in the present case. With the full support of Impella 2.5, a mean blood pressure of 95 $\mathrm{mmHg}$ was required in order to keep the cardiac power output above 0.53 watts, in contrast to the actual mean blood pressure of around $60 \mathrm{mmHg}$ under intensive inotropic sup- port. Based on the concept of curing the heart while maintaining a state of rest in the setting of viral myocarditis, we believe that the Impella device should be considered to be a first-line mechanical circulatory support device for the treatment of fulminant myocarditis with cardiogenic shock.

Due to the absence of a sensitive non-invasive diagnostic modality for detecting viral myocarditis, there are no large population-based, epidemiological studies involving the clinical assessment of viral myocarditis (9). Acute viral myocarditis sometimes revealed a self-limited recovery without any symptoms, while at other times it can cause progressive cardiac damage, leading to features of dilated cardiomyopathy $(1,2)$. In the present case, the left ventricular ejection fraction was $48 \%$ when the patient was discharged after the first hospitalization, a value that was maintained until the present hospitalization. A persistent viral infection in the myocardium may be associated with progressive myocardial dysfunction (9-11), while the incidence of recurrent viral myocarditis is quite low (12).

A total of seven case studies, including the present patient, have reported recurrent viral myocarditis with virus identification (12-17) (Table). Surprisingly, four of these seven patients showed fulmination. Fulminant viral myocarditis is a clinicopathological classification presenting with rapid hemodynamic deterioration, thereby resulting in cardiogenic shock with fatal clinical outcomes. In contrast to the findings of a previous small cohort study (18) reporting 
Table. Case Studies of Recurrent Viral Myocarditis.

\begin{tabular}{lccccccc}
\hline & & \multicolumn{2}{c}{ previous episode(s) } & \multicolumn{2}{c}{ final episode } & & \\
\cline { 3 - 5 } published year (ref.) & sex & age, year & fulminant & age, year & fulminant & type of virus & clinical outcomes \\
\hline $2003(12)$ & male & 75 & no & 75 & yes & Influenza & death \\
$2011(13)$ & female & 29,36 & no, no & 37 & no & Coxsackie & alive \\
$2012(14)$ & female & 5,7 & yes, no & 8 & yes & Influenza & death \\
$2014(15)$ & male & 36,40 & yes, no & 52 & yes & Influenza & alive \\
$2018(16)$ & male & 14 & no & 15 & no & Parvo & alive \\
$2018(17)$ & female & 25,27 & no, no & 28 & no & Erythro & alive \\
our case & male & 34 & yes & 39 & yes & Echo & alive \\
\hline
\end{tabular}

ref.: reference number

a long-term benign prognosis of fulminant myocarditis after the acute critical phase, a recent clinical study indicated worse long-term clinical outcomes with advanced cardiac dysfunction in such cases (19). Experimental studies have proposed several potential mechanisms underlying the pathogenesis of viral myocarditis, in which myocardial injury might be mediated through initial viral toxicity and thereafter the cellular immune response, resulting in persistent viral infection (2). However, precisely why viral myocarditis rarely shows recurrence and/or is occasionally fulminated remains unclear. The three instances of recurrence in four of the seven patients in Table underscore the need to follow the clinical features of the present patient carefully. Future basic and clinical studies must attempt to address these complex issues in order to improve the clinical outcomes of this critical disorder.

In conclusion, we herein report the clinical potential of Impella 5.0 support in the treatment of recurrent fulminant myocarditis with profound cardiogenic shock. Given its effect of cardiac protection with sufficient systemic perfusion, the Impella device should be considered the first-line therapy for the treatment of this critical disorder.

The authors state that they have no Conflict of Interest (COI).

\section{Acknowledgement}

We are deeply indebted to all of the members of the Sakakibara Heart Team for their sincere contributions to caring for the patient, and to Ms. Reina Watanabe for her aid in preparing this article.

\section{References}

1. Kindermann I, Barth C, Mahfoud F, et al. Update on myocarditis. J Am Coll Cardiol 59: 779-792, 2012.

2. Caforio AL, Pankuweit S, Arbustini E, et al. Current state of knowledge on aetiology, diagnosis, management, and therapy of myocarditis: a position statement of the European Society of Cardiology Working Group on Myocardial and Pericardial Diseases. Eur Heart J 34: 2636-2648, 2013.

3. Asaumi Y, Yasuda S, Morii I, et al. Favourable clinical outcome in patients with cardiogenic shock due to fulminant myocarditis supported by percutaneous extracorporeal membrane oxygenation. Eur
Heart J 26: 2185-2192, 2005.

4. Burzotta F, Trani C, Doshi SN, et al. Impella ventricular support in clinical practice: collaborative viewpoint from a European expert user group. Int J Cardiol 201: 684-691, 2015.

5. Fincke R, Hochman JS, Lowe AM, et al. Cardiac power is the strongest hemodynamic correlate of mortality in cardiogenic shock: a report from the SHOCK trial registry. J Am Coll Cardiol 44: 340-348, 2004.

6. Werdan K, Gielen S, Ebelt H, Hochman JS. Mechanical circulatory support in cardiogenic shock. Eur Heart J 35: 156-167, 2014.

7. Nakata J. Hemodynamic support with new percutaneous device (Impella) in patients with cardiogenic shock. The Medical Frontline 71: 1699-1709, 2016.

8. Burkhoff D, Sayer G, Doshi D, Uriel N. Hemodynamics of mechanical circulatory support. J Am Coll Cardiol 66: 2663-2674, 2015.

9. Sagar S, Liu PP, Cooper LT Jr. Myocarditis. Lancet 379: 738-747, 2012.

10. Kühl U, Pauschinger M, Seeberg B, et al. Viral persistence in the myocardium is associated with progressive cardiac dysfunction. Circulation 112: 1965-1970, 2005.

11. Cooper LT Jr. Myocarditis. N Engl J Med 360: 1526-1538, 2009.

12. Takehana $H$, Inomata $T$, Kuwao $S$, et al. Recurrent fulminant viral myocarditis with a short clinical course. Circ J 67: 646-648, 2003.

13. Karavidas A, Lazaros G, Noutsias M, et al. Recurrent coxsackie B viral myocarditis leading to progressive impairment of left ventricular function over 8 years. Int J Cardiol 151: e65-e67, 2011.

14. Lee AF, Chinasson DA, Smythe JF, et al. Recurrent congestive heart failure in a child due to probable myocarditis. Pediatr Cardiol 33: 176-181, 2012.

15. Yoshimizu N, Tominaga $T$, Ito $T$, et al. Repetitive fulminant influenza myocarditis requiring the use of circulatory assist devices. Intern Med 53: 109-114, 2014.

16. Floyd A, Lal A, Molina $\mathrm{K}$, et al. When lightning strikes twice in pediatrics: case report and review of recurrent myocarditis. Pediatrics 141: e20164096, 2018.

17. Nikolaou M, Lazaros G, Karavidas A, Hatzianastasiou S, Miliopoulos D, Adamopoulos S. Recurrent viral myocarditis: the emerging link toward dilated cardiomyopathy. Hellenic J Cardiol 59: 60-63, 2018.

18. McCarthy RE 3rd, Boehmer JP, Hruban RH, et al. Long-term outcome of fulminant myocarditis as compared with acute (nonfulminant) myocarditis. N Engl J Med 342: 690-695, 2000.

19. Ammirati E, Cipriani M, Lilliu M, et al. Survival and left ventricular function changes in fulminant versus nonfulminant acute myocarditis. Circulation 136: 529-545, 2017.

The Internal Medicine is an Open Access journal distributed under the Creative Commons Attribution-NonCommercial-NoDerivatives 4.0 International License. To view the details of this license, please visit (https://creativecommons.org/licenses/ by-nc-nd/4.0/).

(C) 2019 The Japanese Society of Internal Medicine Intern Med 58: 1459-1462, 2019 